\title{
Ocorrência de carrapatos em tamanduá-bandeira (Myrmecophaga tridactyla) e tamanduá- mirim (Tamandua tetradactyla) na região do Pantanal Sul Mato-Grossense, Brasil
}

\author{
Occurence of ticks on giant anteater (myrmecophaga tridactyla and collared anteater (tamandua \\ tetradactyla) in the pantanal region of Mato Grosso do Sul State, Brazil
}

\author{
João Ricardo Martins ${ }^{1}$ Ísis Meri Medri $^{2}$ Carlos Marcos Oliveira ${ }^{3}$ Alberto Guglielmone ${ }^{4}$
}

\section{- NOTA -}

\section{RESUMO}

Registra-se a ocorrência de carrapatos em tamanduás-bandeira e tamanduás-mirim na região do pantanal sul mato-grossense entre os meses de março e novembro de 2001. As espécies identificadas foram Amblyomma cajennense (123 machos e 63 fêmeas), A. parvum (35 machos, 67 fêmeas) e A. nodosum (2 machos), um parasita específico de tamanduás em sua fase adulta. De um total de 20 tamanduás examinados no período de estudo, A. cajennense foi encontrado na maioria dos animais (15), seguido por $\boldsymbol{A}$. parvum (9) e por A. nodosum (2).

Palavras-chave: Amblyomma, carrapatos, tamanduá-bandeira, tamanduá-mirim, Pantanal.

\section{ABSTRACT}

The occurrence of ticks on anteaters in the Pantanal region, Mato Grosso do Sul state, Brazil, between March and February 2001 is described. Tick species identified were Amblyomma cajennense (123 males and 63 females), A. parvum (35 males, 67 females) and A. nodosum (2 males), species considered as specific of anteater in its adult phase. A. cajennense was found in the majority of animals (15) followed by A. parvum (9) and by A. nodosum (2) from a total of 20 anteaters examined in the study period.

Key words: Amblyomma, collared anteater, giant anteater, Ixodidae, Pantanal, ticks.

Uma das preocupações com a identificação e o registro de algumas espécies de carrapatos em animais silvestres está relacionada aos riscos potenciais de que estes artrópodes podem transmitir patógenos a outros animais silvestres e domésticos, bem como aos humanos. Na região do Pantanal, convivem diversas espécies de animais silvestres e, nos últimos anos, registrou-se um incremento na população bovina e humana. Observações iniciais da presença desses artrópodes em animais silvestres no Pantanal foram relatadas no início do século passado (ARAGÃO, 1936; ARAGÃO \& FONSECA, 1961) e mais recentemente, foram identificadas espécies de carrapatos na fauna silvestre da região (ITO et al., 1998; PEREIRA et al., 2000). Este trabalho relata as espécies de carrapatos encontradas em tamanduás no Pantanal Sul MatoGrossense, entre os meses de março e novembro de 2001, numa tentativa de contribuir para futuros estudos epidemiológicos visando esclarecer as interações entre os hospedeiros e os seus ectoparasitas.

Os carrapatos foram colhidos de tamanduás capturados entre os meses de março e novembro de 2001 nas localidades do Pantanal da Nhecolândia e Pantanal do Rio Negro, Estado do Mato Grosso do Sul. Os espécimens foram colocados em frascos identificados, contendo álcool $70^{\circ}$, e enviados ao Centro de Pesquisa Veterinária Desidério Finamor (CPVDF), Eldorado do Sul, RS. Para identificação preliminar, foi utilizada a chave de ARAGÃO \& FONSECA (1961), sendo que alguns exemplares foram confirmados no laboratório de Entomozooses da Faculdade de Veterinária da UFRGS, Porto Alegre, RS, e o restante, foi identificado por um dos autores (A. A. Guglielmone) no Instituto Nacional de Tecnologia Agropecuária (INTA), Rafaela, Argentina.

A tabela 1 apresenta os resultados das identificações realizadas nos 20 tamanduás examinados, com

${ }^{1}$ Médico Veterinário, MSc, FEPAGRO - Centro de Pesquisa Veterinária Desidério Finamor. Estrada do Conde, 6000, 92990-000,

Eldorado do Sul, RS. Doutorando em Ciência Animal pela UFMG. E-mail: joaorsm@zaz.com.br

${ }^{2}$ Biólogo, MSc, Embrapa - Pantanal, Caixa Postal 109, 79320-900, Corumbá, MS.

${ }^{3}$ Médico Veterinário, PhD, Faculdade de Veterinária da UFRGS, Avenida Bento Gonçalves, 9090, Porto Alegre,RS

${ }^{4}$ Médico Veterinário, PhD, EEA Rafaela, Santa Fé, CC22, 2300. Argentina 
Tabela 1 - Lista de carrapatos identificados em tamanduás na região do pantanal sul mato-grossense.

\begin{tabular}{|c|c|c|c|}
\hline $\begin{array}{l}\text { Data da colheita } \\
\left(\mathbf{N}^{\circ} \text { registro) }\right.\end{array}$ & Local & Hospedeiro & Espécie identificada (M/F/N) \\
\hline 17/03/01 (182) & Nhecolândia, MS & Tamanduá-bandeira & A. cajennense $(15 \mathrm{M} ; 08 \mathrm{~F})$ \\
\hline 29/03/01 (183) & Faz. Nhumirim,MS & Tamanduá-bandeira & A. cajennense $(25 \mathrm{M} ; 23 \mathrm{~F})$ \\
\hline $31 / 03 / 01$ & Faz. Porto Alegre,MS & Tamanduá-bandeira & A. cajennense $(16 \mathrm{M} ; 4 \mathrm{~F})$ \\
\hline 04/04/01 (193) & Faz. Nhumirim, MS & Tamanduá-bandeira & $\begin{array}{c}\text { A. cajennense }(8 \mathrm{M} ; 3 \mathrm{~F}) \\
\text { A. parvum }(14 \mathrm{M} ; 3 \mathrm{~F})\end{array}$ \\
\hline 23/04/01 & Faz. Porto Alegre,MS & Tamanduá-bandeira & A. cajennense $(12 \mathrm{M} ; 1 \mathrm{~F})$ \\
\hline $25 / 04 / 01$ & Faz. Nhumirim,MS & Tamanduá-bandeira & A. cajennense $(21 \mathrm{M} ; 5 \mathrm{~F})$ \\
\hline $24 / 05 / 01$ & Faz. Porto Alegre,MS & Tamanduá-bandeira & A. cajennense $(5 \mathrm{M} ; 4 \mathrm{~F})$ \\
\hline 25/05/01 (188) & Faz. Nhumirim,MS & Tamanduá-bandeira & A. cajennense $(5 \mathrm{M} ; 6 \mathrm{~F})$ \\
\hline $25 / 05 / 01$ & Faz. Porto Alegre,MS & Tamanduá-bandeira & A. cajennense $(7 \mathrm{M} ; 1 \mathrm{~F})$ \\
\hline 09/03/01 (190) & & Tamanduá-bandeira & A. cajennense (1F) \\
\hline 24/03/01(192) & BR-262,MS & Tamanduá-mirim & A. nodosum (1M) \\
\hline 04/07/01 (193) & Faz. Nhumirim, MS & Tamanduá-bandeira & $\begin{array}{c}\text { A. parvum }(3 \mathrm{~F}, 4 \mathrm{M}) \\
\text { A. cajennense }(3 \mathrm{~F}, 8 \mathrm{M})\end{array}$ \\
\hline 04/07/01 (194) & Faz. Rio Negro, MS & Tamanduá-bandeira & A. parvum (4F) \\
\hline 04/07/01 (195) & Faz. Rio Negro, MS & Tamanduá-bandeira & $\begin{array}{c}\text { A. parvum }(2 \mathrm{~F}) \\
\text { A. cajennense }(5 \mathrm{M})\end{array}$ \\
\hline 06/07/01 (196) & Faz. Rio Negro, MS & Tamanduá-bandeira & $\begin{array}{c}\text { A. parvum }(3 \mathrm{~F}) \\
\text { A. nodosum }(1 \mathrm{M})\end{array}$ \\
\hline 18/07/01 (197) & Faz. Nhumirim, MS & Tamanduá-mirim & A. parvum (6F) \\
\hline 23/07/01 (198) & Faz. Nhumirim, MS & Tamanduá-bandeira & A. parvum $(2 \mathrm{M} ; 21 \mathrm{~F})$ \\
\hline 24/07/01 (199) & Faz. Nhumirim, MS & Tamanduá-bandeira & $\begin{array}{l}\text { A. cajennense }(2 \mathrm{M} ; 1 \mathrm{~F}) \\
\text { A. parvum }(1 \mathrm{M} ; 12 \mathrm{~F})\end{array}$ \\
\hline 25/07/01 (200) & Faz. Nhumirim, MS & Tamanduá-bandeira & $\begin{array}{l}\text { A. cajennense }(1 \mathrm{M}) \\
\text { A. parvum }(12 \mathrm{~F})\end{array}$ \\
\hline 20/09/01 (206) & Faz. Nhumirim, MS & Tamanduá-bandeira & A. parvum (4F) \\
\hline 22/11/01 (207) & Faz. Nhumirim, MS & Tamanduá-bandeira & A. cajennense (3F) \\
\hline 22/11/01 (208) & Faz. Nhumirim, MS & Tamanduá-bandeira & A. cajennense $(3 \mathrm{~F})$ \\
\hline
\end{tabular}

M - machos

F - fêmeas

os locais das colheitas, datas e espécies de carrapatos encontradas. De acordo com os resultados obtidos , a presença de Amblyomma cajennense foi observada na maioria dos animais examinados (15), seguido pelo A. parvum (9), sendo que o $\boldsymbol{A}$. nodosum foi registrado em 2 dos 20 tamanduás examinados no período. A. cajennense foi a espécie também encontrada em maior número em todas as colheitas realizadas. Os resultados evidenciam a baixa especificidade desta espécie resultando em um alto potencial de sobrevivência. A. nodosum em sua fase adulta, tem sido encontrado quase que exclusivamente em tamanduás (GUIMARÃES et al., 2001) enquanto que o A. parvum é encontrado em várias espécies de hospedeiros, inclusive em humanos. Uma redescrição deste ixodídeo foi feita na Argentina, onde é um parasita comum do gado no norte daquele país (GUGLIELMONE et al., 1990).
Há relatos da presença deste carrapato em diversas espécies silvestres de vários estados brasileiros (GUIMARÃES et al., 2001) enquanto com relação à ocorrência sobre tamanduás na região estudada, não foi encontrada referência de parasitismo. A presença de A. cajennense foi descrita em capivaras (Hydrochaeris hydrochaeris) e queixada (Tayassu pecari), na região do pantanal (ITO et al., 1998), sendo que também o carrapato Boophilus microplus foi identificado sobre queixada, alertando que os animais domésticos introduzidos na região podem estar contribuindo para a disseminação de carrapatos entre os animais silvestres. $\mathrm{O}$ fato de A cajennense não apresentar especificidade em relação aos hospedeiros pode propiciar maiores riscos de disseminação de patógenos transmitidos por estes artrópodes com o envolvimento humano. 


\section{REFERÊNCIAS BIBLIOGRÁFICAS}

ARAGÃO, H. Ixodidas brasileiros e de alguns paizes limitrophes. Memórias do Instituto Oswaldo Cruz, v. 31, n. 4, p. 759-844, 1936.

ARAGÃO, H.; FONSECA, F. Notas de Ixodologia. VIII.Lista e chave para os representantes da fauna ixodológica brasileira. Memórias do Instituto Oswaldo Cruz, v.59, n.2, p.115-129, 1961.

ITO, F.H. et al. Evidência sorológica de brucelose e leptospirose e parasitismo por ixodídeos em animais silvestres do pantanal Sul-Mato-Grossense. ARS Veterinária, v.14, n.3, p.302-310, 1998 .
GUGLIELMONE, A.A.; MANGOLD, A.J.; KEIRANS, J. Redescription of the male and female of Amblyomma parvum Aragão, 1908, and description of the nymph and larva, and description of all stages of Amblyomma pseudoparvum sp.N. (Acari: Ixodida: Ixodidae). Acarology, v. 31, n.2, p.143159, 1990.

GUIMARÃES, J.C.; TUCCI, E.C.; BARROS-BATESTTI, D M. Ectoparasitos de importância veterinária. São Paulo: Plêiade, 2001. 213p.

PEREIRA, M.C. et al. Ticks (Acari:Ixodidae) associated with wild animals in the pantanal region of Brazil. Journal of Medical Entomology, v.37, n.6, p.979983,2000 . 